\title{
Using Bayesian Belief Networks and Fuzzy Logic to Evaluate Aquatic Ecological Risk
}

\author{
Kevin Fong-Rey Liu, Ken Yeh, Cheng-Wu Chen, Han-Hsi Liang, and Yung-Shuen Shen
}

\begin{abstract}
There are three issues: 1) ecological effects quotient is a low-level toxicity risk estimate for chemical pressure because it uses a simple number to represent a complex biological problem; 2) besides biological toxicity, species future status is also relevant to physical and biological pressures which are difficult to express by ecological effects quotient; 3) we need a higher level methodology to consider all ecological pressures. To address the issues, this study has five objectives: 1) to design the ecological risk procedure for Bayesian belief networks (BBN) approach; 2) to represent the relationships between physical, chemical and biological pressures and effects with $\mathrm{BBN}$; 3) to construct conditional probabilities in BBN by fuzzy logic; and 4) study case.
\end{abstract}

Index Terms-Bayesian belief networks, fuzzy logic, aquatic ecological risk.

\section{INTRODUCTION}

The rivers located in the south part of Taiwan are usually severe polluted. This study focuses on the four rivers as the case studies: Chiang-Chun River, Erh-Jen River, Gao-Ping River and Dong-Gang River, as shown in Fig. 1. According the related work [1]-[4], the most vulnerable species is Cyprinus carpio, whose future population status represents the condition of the aquatic ecological risk in this study.

This study attempts to use Bayesian belief networks (BBN) to model the probabilistic cause-and-effect dependencies between the factors affecting future population status of Cyprinus carpio. Several studies have demonstrated the utility of BBN in garnering and integrating expert knowledge and empirical data concerning ecological issues. The BBN is a directed acyclic graph with nodes that denotes a set of random variables as nodes and arrows, which indicate their probabilistic cause-and-effect dependencies. A number of properties make it particularly useful in ecological applications; it allows a combination of qualitative knowledge and quantitative data, so it can easily cope with missing data and it can be constructed using observed data, other models, or expert knowledge. It is a useful tool for risk assessment, because it explicitly incorporates uncertainty in

Manuscript received January 13, 2013; revised March 13, 2013.

K. F. R. Liu (Corresponding author) is with the Department of Safety, Health and Environmental Engineering, Ming Chi University of Technology, New Taipei City, Taiwan (e-mail: kevinliu@mail.mcut.edu.tw).

K. Yeh is with the Department of Construction Science and Technology, De-Lin Institute of Technology, New Taipei City, Taiwan (e-mail: kyeh@dlit.edu.tw).

C. W. Chen is with the Institute of Maritime Information and Technology, National Kaohsiung Marine University, Kaohsiung, Taiwan (e-mail: chengwu@webmail.nkmu.edu.tw).

H. H. Liang is with the Department of Architecture, National United University, Miaoli, Taiwan (e-mail: 1hh@nuu.edu.tw).

Y. S. Shen is with the Holistic Education Center, Mackay Medical College, New Taipei City, Taiwan (e-mail: ysshen@mmc.edu.tw). relationships. It is also useful in ecological risk management, because identifies the effects, given the causes, or the causes, given the effects.

This study also aims to use fuzzy logic [5] as a systematic method to help experts generate conditional probabilities (CP).

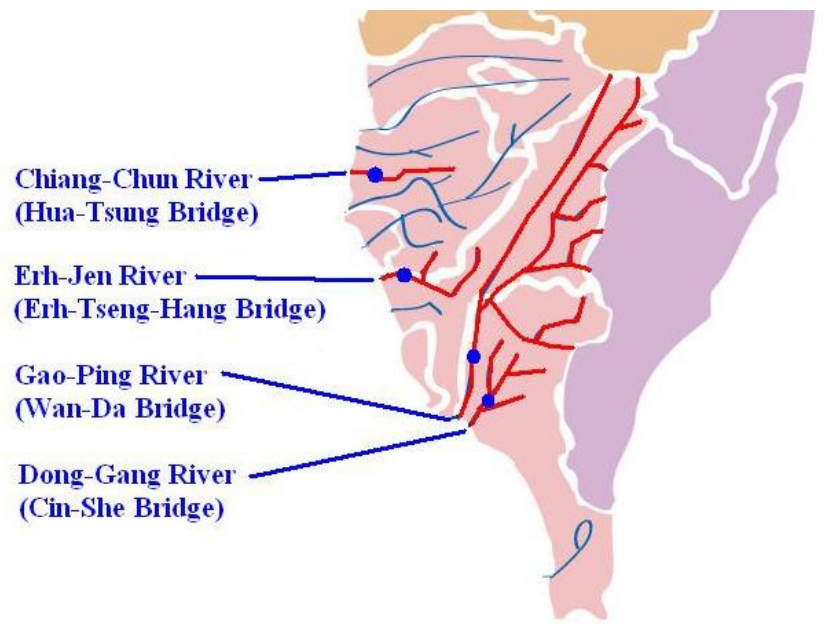

Fig. 1. Magnetization as a function of applied field.

\section{Methods AND MATERIALS}

\section{A. Ecological Risk Assessment}

The overall ecological risk assessment process [6] includes problem formulation, ecological response analysis and exposure analysis (Fig. 2). In problem formulation, the purpose for the assessment is articulated, the problem is defined, and a plan for analyzing and characterizing risk is determined. The ecological response analysis analyzes effects by examining stressor-response relationships, the evidence for causality, and the relationship between measures of effect and assessment endpoints. The exposure analysis analyzes exposure by examining the sources of stressors, the distribution of stressors in the environment, and the extent of co-occurrence or contact.

\section{B. Bayesian Belief Networks}

Bayesian belief networks (BBN) [7] are very effective for modeling situations where some information is already known and incoming data is uncertain or partially unavailable. Uncertainty arises in many situations. For example, experts may be uncertain about their own knowledge, there may be uncertainty inherent in the situation being modeled, or uncertainty about the accuracy and availability of information. Because BBN offer consistent semantics for representing uncertainty and an intuitive graphical representation of the interactions between various causes and effects, they are a very effective method of 
modeling uncertain situations that depend on cause and effect.

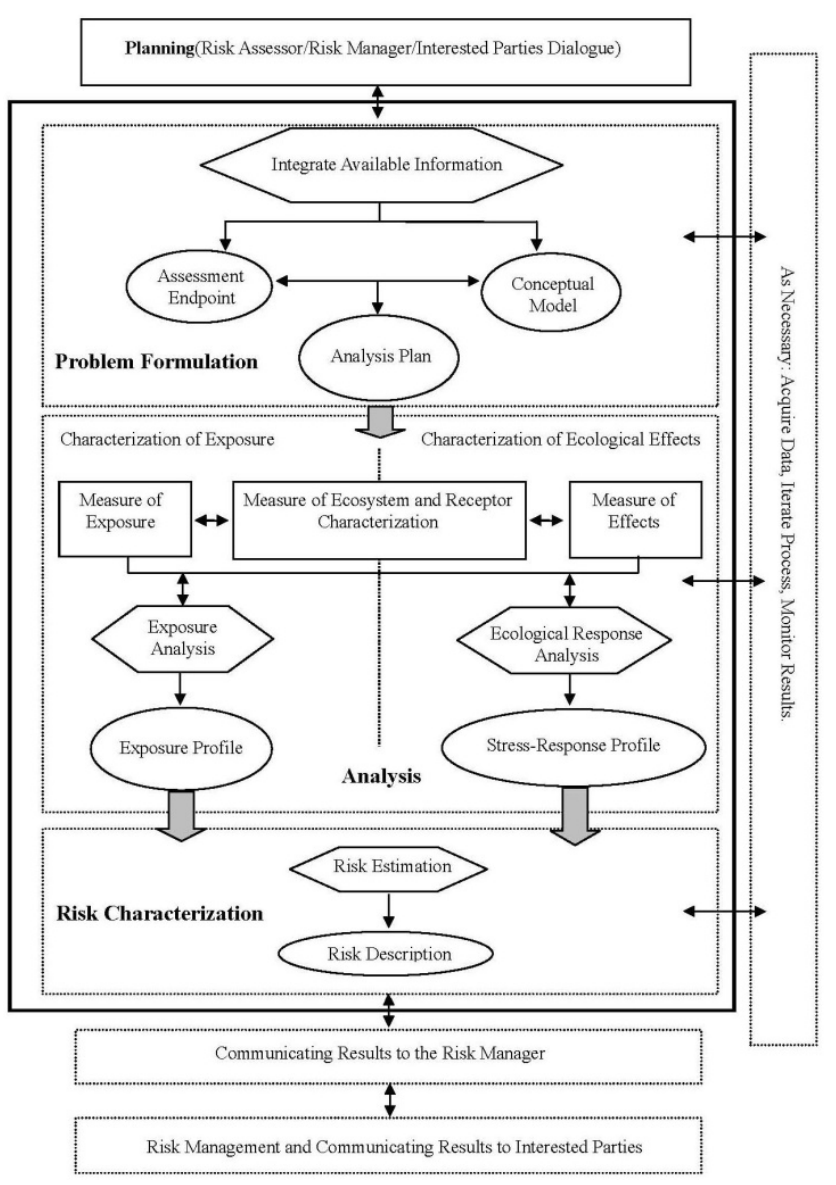

Fig. 2. Magnetization as a function of applied field.

Each of the variables in the Bayesian belief network is represented by nodes. A variable in a belief network could be whether a light switch is on, the proximity of an enemy battalion, or the RPM of an engine. Each node has states, or a set of probable values for each variable. For example, the weather could be cloudy or sunny, an enemy battalion could be near or far, symptoms present or not present, and the garbage disposal working or not working. Nodes are connected to show causality with an arrow indicating the direction of influence. These arrows are called edges.

A Bayesian belief network is a model that represents the possible states of a given domain. A Bayesian belief network also contains probabilistic relationships among some of the states of the domain. The probability of any node in the Bayesian belief network being in one state or another without current evidence is described using a conditional probability table. Probabilities on some nodes are affected by the state of other nodes, depending on causality. Prior information about the relationships among nodes may indicate that the likelihood that a node is in one state is dependent on another node's state. With the historical information stored in the conditional probability tables, BBN can be used to help make decisions, or as a way of automating a decision-making process.

\section{Fuzzy Logic}

A fuzzy expert system [5] is an expert system that uses a collection of fuzzy membership functions and rules, instead of Boolean logic, to reason about data. The rules in a fuzzy expert system are usually of a form similar to the following:

If $\mathrm{x}$ is low and $\mathrm{y}$ is high then $\mathrm{z}=$ medium

where $\mathrm{x}$ and $\mathrm{y}$ are input variables (names for know data values), $\mathrm{z}$ is an output variable (a name for a data value to be computed), low is a membership function (fuzzy subset) defined on $\mathrm{x}$, high is a membership function defined on $\mathrm{y}$, and medium is a membership function defined on $z$. The antecedent (the rule's premise) describes to what degree the rule applies, while the conclusion (the rule's consequent) assigns a membership function to each of one or more output variables. Most tools for working with fuzzy expert systems allow more than one conclusion per rule. The set of rules in a fuzzy expert system is known as the rulebase or knowledge base. The general inference process proceeds in three (or four) steps.

1. Under FUZZIFICATION, the membership functions defined on the input variables are applied to their actual values, to determine the degree of truth for each rule premise.

2. Under INFERENCE, the truth value for the premise of each rule is computed, and applied to the conclusion part of each rule. This results in one fuzzy subset to be assigned to each output variable for each rule.

3. Under COMPOSITION, all of the fuzzy subsets assigned to each output variable are combined together to form a single fuzzy subset for each output variable.

4. Finally is the (optional) DEFUZZIFICATION, which is used when it is useful to convert the fuzzy output set to a crisp number. In the CENTROID method, the crisp value of the output variable is computed by finding the variable value of the center of gravity of the membership function for the fuzzy value.

\section{RESULTS}

\section{A. Problem Formulation}

TABLE I: GRADES OF FACTORS AND THEIR DESCIPTION

\begin{tabular}{cccccc}
\hline \hline Factor & \multicolumn{5}{c}{ Grade and its definition } \\
\hline Temperature & $0-8$ & $9-16$ & $17-23$ & $24-32$ & $>33$ \\
Flow Velocity & $0-0.1$ & $0.11-0.2$ & $0.21-1$ & $1.1-2$ & $2.1-3$ \\
Mud & 1 & 2 & 3 & 4 & 5 \\
Water Depth & $0-0.25$ & $0.26-0.5$ & $0.51-10$ & $11-20$ & $21-30$ \\
DO & $0-5$ & $6-10$ & $11-17$ & $18-25$ & $26-30$ \\
PH & $0-3$ & $4-6.5$ & $6.5-9$ & $9.1-12$ & $13-14$ \\
Aquatic Vegetation & 1 & 2 & 3 & 4 & 5 \\
Zooplankton & 1 & 2 & 3 & 4 & 5 \\
Protozoans & 1 & 2 & 3 & 4 & 5 \\
Small Crustaceans & 1 & 2 & 3 & 4 & 5 \\
Insect Larva & 1 & 2 & 3 & 4 & 5 \\
$\mathrm{Hg}$ & $<0.1$ & $0.11-0.5$ & $0.51-1$ & $1-10$ & $>10$ \\
$\mathrm{~Pb}$ & $<0.1$ & $0.11-0.5$ & $0.51-1$ & $1-10$ & $>10$ \\
$\mathrm{Cu}$ & $<0.1$ & $0.11-0.5$ & $0.51-1$ & $1-10$ & $>10$ \\
$\mathrm{Zn}$ & $<0.1$ & $0.11-0.5$ & $0.51-1$ & $1-10$ & $>10$ \\
$\mathrm{Cd}$ & $<0.1$ & $0.11-0.5$ & $0.51-1$ & $1-10$ & $>10$ \\
$\mathrm{NH}$-N & $<0.1$ & $0.11-0.5$ & $0.51-1$ & $1-10$ & $>10$ \\
Exterior Threat & 1 & 2 & 3 & 4 & 5 \\
\hline \hline
\end{tabular}

In accordance with the literature, several essential survival factors influencing the future population status of the Cyprinus carpio are shown in Fig. 3. The factors include 
spawning, habitat, diet and toxicity and their sub-factors are. temperature, flow Velocity, mud, water depth, DO, PH, aquatic vegetation, zooplankton, protozoans, Small crustaceans, insect larva, $\mathrm{Hg}, \mathrm{Pb}, \mathrm{Cu}, \mathrm{Zn}, \mathrm{Cd}, \mathrm{NH}_{3}-\mathrm{N}$ and exterior threat. Their assessment grades and the associated definitions are shown in Table I.

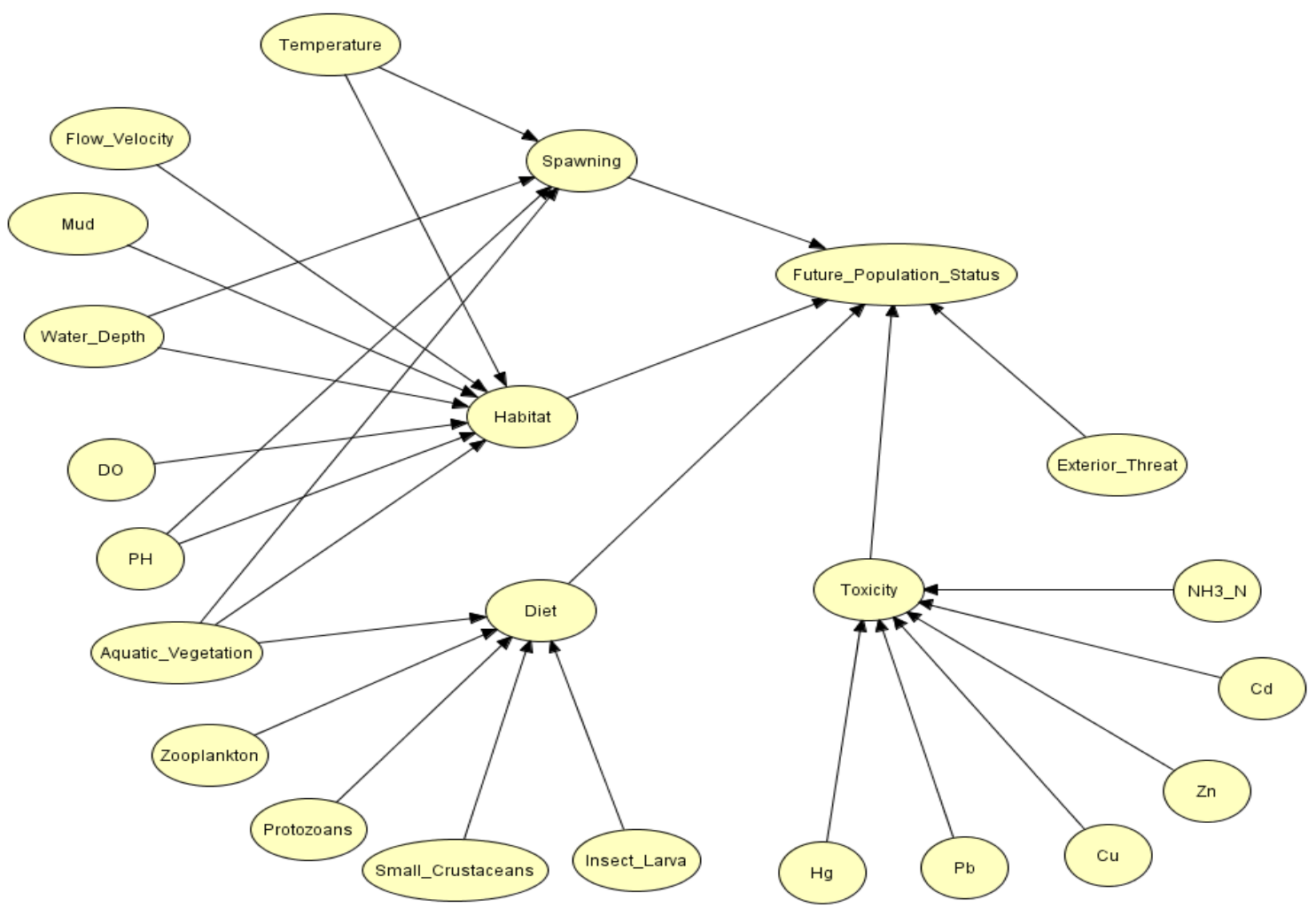

Fig. 3. Essential survival factors influencing the future population status of the Cyprinus carpio
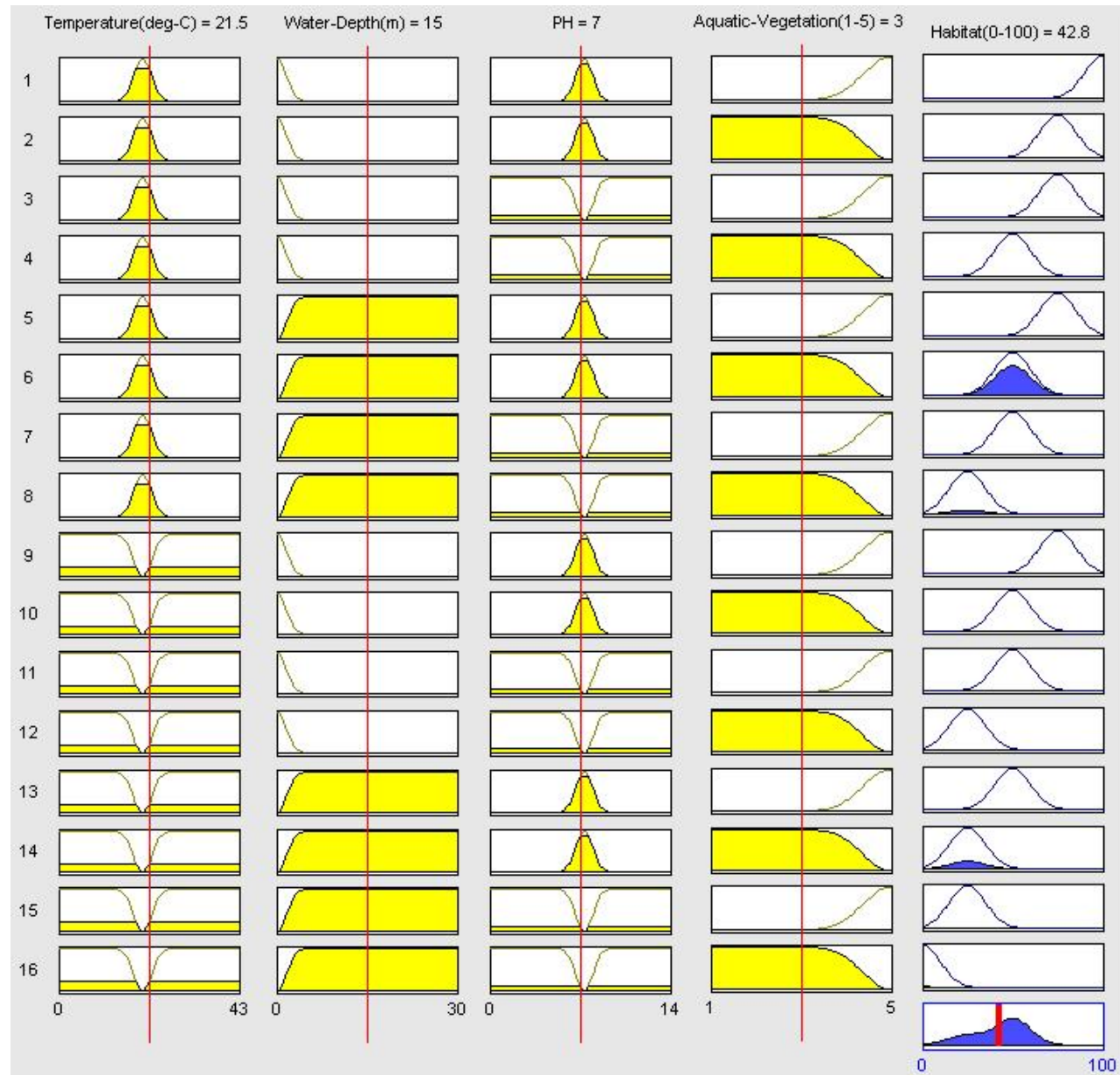

Fig. 4. The reasoning process to generate CPs. 


\section{B. Ecological Response Analysis}

In this paper, there is difficulty in determining CPs because of the lack of literature on probabilistic relationships between causes and effects and so subjective judgment is exploited. The BBN model in this study contains 78,625 CPs, so expert evaluation is a very difficult task. Fuzzy logic is used to help the experts to generate the CPs. For example, the reasoning process to generate $\mathrm{CPs}$, for evaluating habitat, is shown in Fig. 4, and its memberships functions about the fuzzy terms used in this systems are also defined in this figure.

\section{Exposure Analysis}

This study selects the appropriate points for assessing the four rives and they are Hua-Tsung Bridge for Chiang-Chun River (Fig. 5), Erh-Tseng-Hang Bridge for Erh-Jen River(Fig 6), Wan-Da Bridge for Gao-Ping River (Fig. 7) and Cin-She Bridge for Dong-Gang River (Fig. 8). The input of these rivers for the $\mathrm{BBN}$ model is evaluated at these points, as shown in Table II.

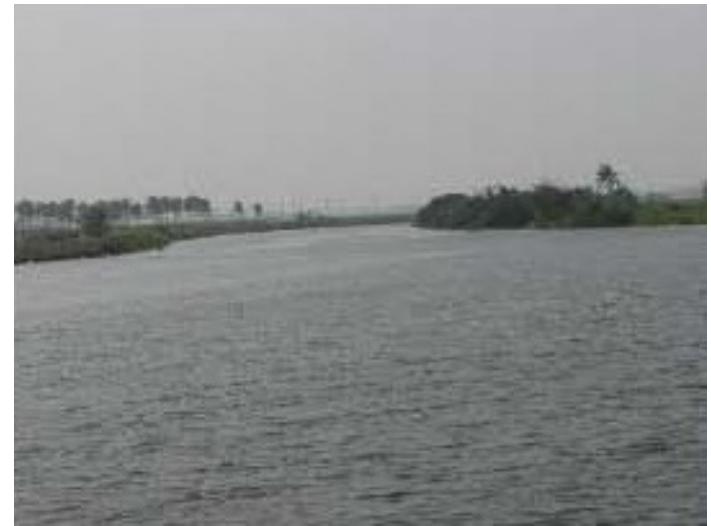

Fig. 5. Chiang-Chun River (Hua-Tsung bridge)[1-4].

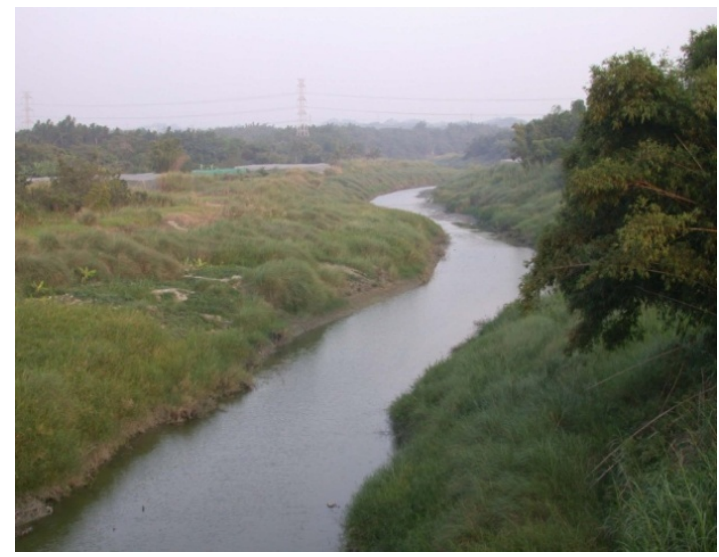

Fig. 6. Erh-Jen River (Erh-Tseng-Hang Bridge) [1-4].

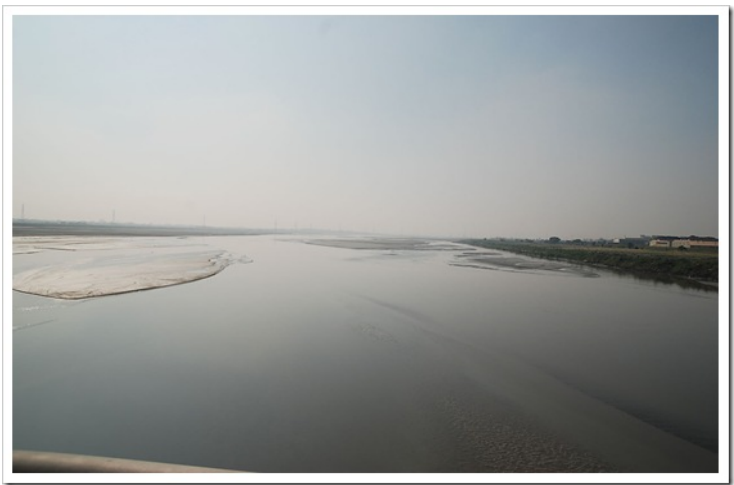

Fig. 7. Gao-Ping River (Wan-Da Bridge) [1-4]

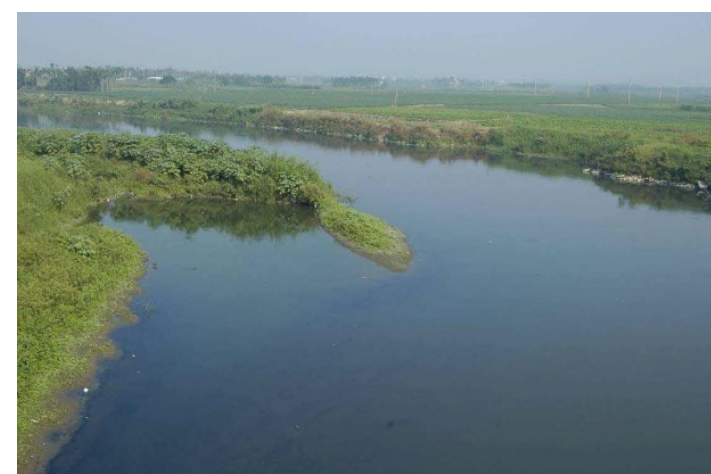

Fig. 8. Dong-Gang River (Cin-She Bridge) [1-4].

TABLE II: THE INPUT FOR THE BBN MODEL

\begin{tabular}{ccccc}
\hline \hline Factor & $\begin{array}{c}\text { Chiang-Chun } \\
\text { River (Hua-Tsung } \\
\text { Bridge) }\end{array}$ & $\begin{array}{c}\text { Erh-Jen River } \\
\text { (Erh-Tseng-Hang } \\
\text { Bridge) }\end{array}$ & $\begin{array}{c}\text { Gao-Ping River } \\
\text { (Wan-Da Bridge) }\end{array}$ & $\begin{array}{c}\text { Dong-Gang River } \\
\text { (Cin-She Bridge) }\end{array}$ \\
\hline Temperature & 3 & 3 & 3 & 3 \\
Flow Velocity & 3 & 3 & 4 & 2 \\
Mud & 4 & 4 & 2 & 3 \\
Water Depth & 3 & 3 & 4 & 3 \\
DO & 1 & 1 & 1 & 1 \\
PH & 3 & 3 & 3 & 3 \\
Aquatic Vegetation & 4 & 4 & 3 & 3 \\
Zooplankton & 4 & 4 & 4 & 2 \\
Protozoans & 3 & 4 & 4 & 2 \\
Small Crustaceans & 3 & 3 & 3 & 2 \\
Insect Larva & 2 & 3 & 3 & 2 \\
$\mathrm{Hg}$ & 1 & 1 & 1 & 1 \\
$\mathrm{~Pb}$ & 1 & 1 & 1 & 1 \\
$\mathrm{Cu}$ & 2 & 1 & 1 & 1 \\
$\mathrm{Zn}$ & 2 & 4 & 3 & 1 \\
$\mathrm{Cd}$ & 1 & 1 & 1 & 1 \\
$\mathrm{NH}$-N & 5 & 5 & 5 & 5 \\
Exterior Threat & 4 & 3 & 3 & 2 \\
\hline \hline
\end{tabular}

\section{Risk Characterization}

The prediction of the future population status of the Cyprinus carpio in the four rives is carried out by the BBN model. The BBN results of the Hua-Tsung Bridge for Chiang-Chun River and the Erh-Tseng-Hang Bridge for Erh-Jen River are shown in from Figs. 9 and 10, respectively. The all results are also summarized in Table III.

\section{DISCUSSION}

Sometimes it is useful to know the degree to which a belief in a particular node is influenced by findings at other nodes. Hugin computes a node's 'value of information', using the mutual information. In probability theory and information theory, the mutual information of two random variables is a quantity that measures the mutual dependence of the two random variables. The mutual information determines which variables and states of variables are more influential, with respect to the target variable. It shows when small changes in the probability of a state cause great changes in the probability distribution of the target variable. The information also helps to identify errors in either the network structure or the CPs and provides guidance for the collection of further data or for eliciting direct expert evaluation. For the case study, the result for the mutual information is shown in Fig. 11. For the target node (future population status), toxicity, exterior threat, the suitability of spawning are the most crucial survival factors. 


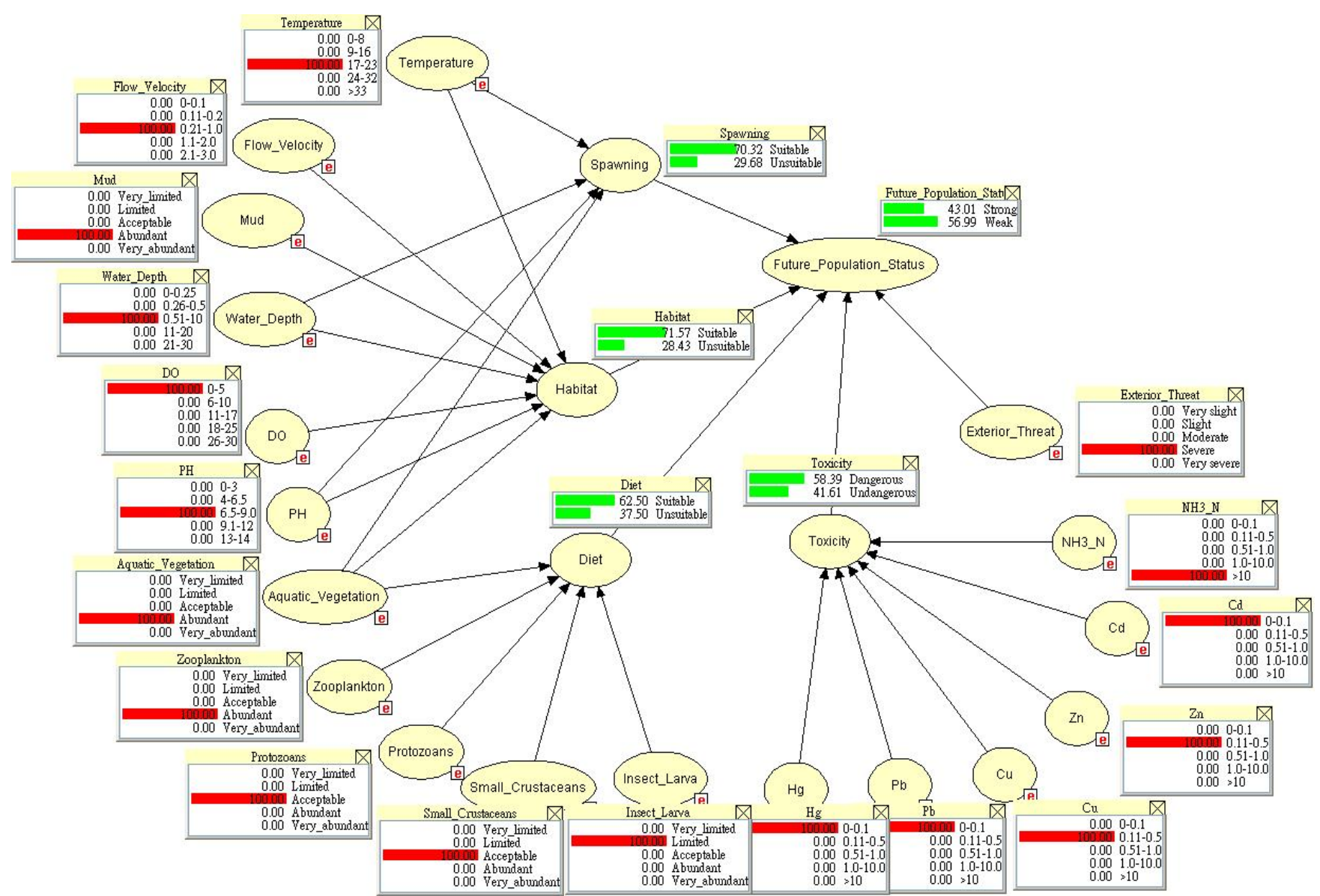

Fig. 9. The BBN result of Chiang-Chun River (Hua-Tsung bridge).

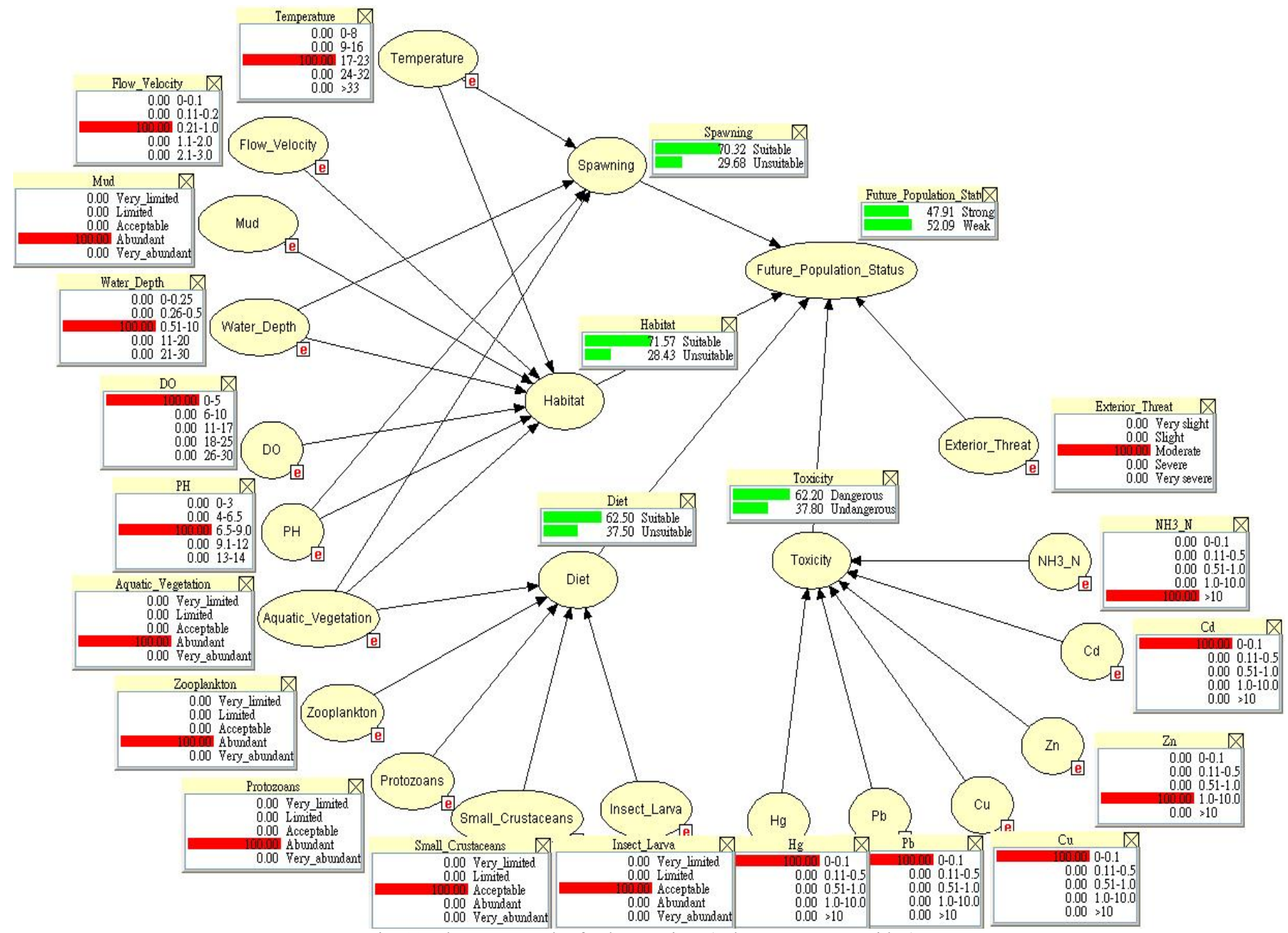

Fig. 10. The BBN result of Erh-Jen River (Erh-Tseng-Hang Bridge). 
TABLE III: THE PREDiction OF THE FUtURE PopUlation STATUS OF THE CYPRINUS CARPIO USING BBN

\begin{tabular}{ccccc}
\hline \hline \multirow{2}{*}{ Factor } & \multicolumn{2}{c}{$\begin{array}{c}\text { Chiang-Chun River } \\
\text { (Hua-Tsung Bridge) }\end{array}$} & \multicolumn{2}{c}{$\begin{array}{c}\text { Erh-Jen River } \\
\text { (Erh-Tseng-Hang Bridge) }\end{array}$} \\
\cline { 2 - 5 } & Suitable & Unsuitable & Suitable & Unsuitable \\
\hline Spawning & $70.32 \%$ & $29.68 \%$ & $70.32 \%$ & $29.68 \%$ \\
Habitat & $71.57 \%$ & $28.43 \%$ & $71.57 \%$ & $28.43 \%$ \\
Diet & $62.50 \%$ & $37.50 \%$ & $62.50 \%$ & $37.50 \%$ \\
\cline { 2 - 5 } Toxicity & Dangerous & Undangerous & Dangerous & Undangerous \\
\cline { 2 - 5 } & $58.39 \%$ & $41.61 \%$ & $62.20 \%$ & $37.80 \%$ \\
\hline Future Population & Strong & Weak & Strong & Weak \\
\cline { 2 - 5 } Status & $43.01 \%$ & $56.99 \%$ & $47.91 \%$ & $52.09 \%$ \\
\hline \hline
\end{tabular}

TABLE III: The PREDiction OF THE FUtURe Population Status of the CYPRINUS CARPIO USING BBN (CONTINUED)

\begin{tabular}{ccccc}
\hline \hline \multirow{2}{*}{ Factor } & \multicolumn{2}{c}{$\begin{array}{c}\text { Gao-Ping River } \\
\text { (Wan-Da Bridge) }\end{array}$} & \multicolumn{2}{c}{$\begin{array}{c}\text { Dong-Gang River } \\
\text { (Cin-She Bridge) }\end{array}$} \\
\cline { 2 - 5 } & Suitable & Unsuitable & Suitable & Unsuitable \\
\hline Spawning & $44.97 \%$ & $55.03 \%$ & $67.74 \%$ & $32.26 \%$ \\
Habitat & $38.24 \%$ & $61.76 \%$ & $64.30 \%$ & $35.70 \%$ \\
Diet & $62.50 \%$ & $37.50 \%$ & $33.00 \%$ & $67.00 \%$ \\
\cline { 2 - 5 } Toxicity & Dangerous & Undangerous & Dangerous & Undangerous \\
\cline { 2 - 5 } & $58.60 \%$ & $41.40 \%$ & $58.05 \%$ & $41.95 \%$ \\
\hline Future Population & Strong & Weak & Strong & Weak \\
\cline { 2 - 5 } Status & $43.48 \%$ & $56.52 \%$ & $52.97 \%$ & $47.03 \%$ \\
\hline \hline
\end{tabular}

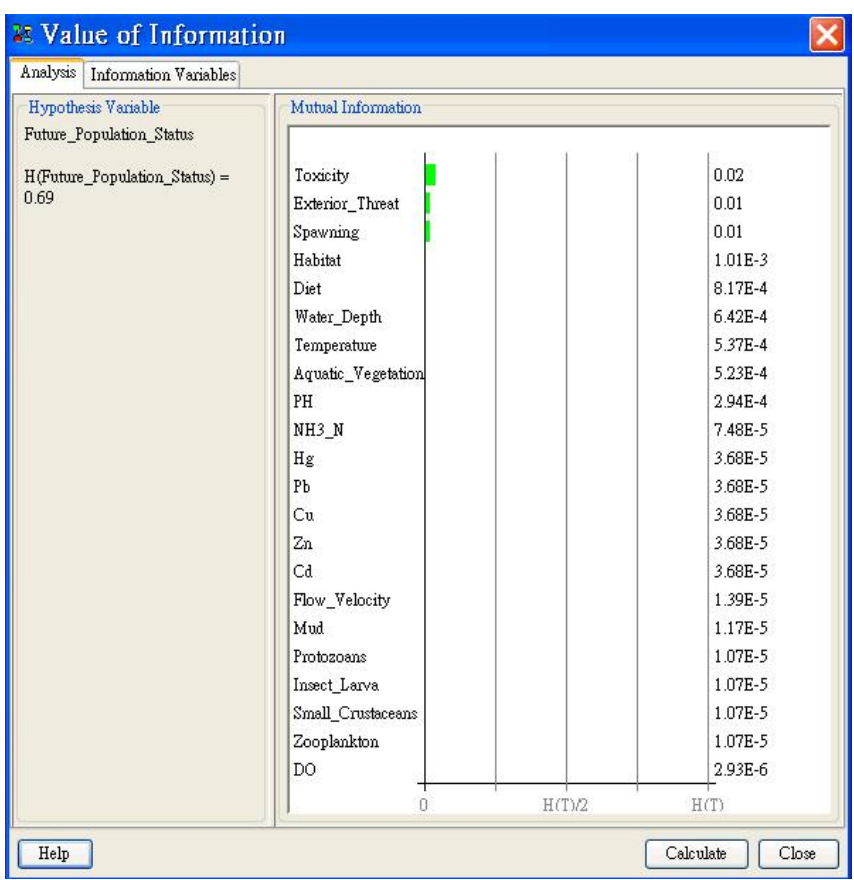

Fig. 11. Magnetization as a function of applied field.

\section{CONCLUSION}

This study proposes a BBN model for aquatic ecological assessment, with features including the representation of the probabilistic relationships between survival factors and adverse ecological effects using the graphical structures of the $\mathrm{BBN}$, the construction of stress-response relationships using the $\mathrm{CPs}$ of the $\mathrm{BBN}$ and the capability of predicting the population status of a rare animal using the inference mechanism of the BBN. The 78,625 conditional probabilities in the BBN model are very difficult to generate, because of the lack of sufficient data concerning rare animals. Therefore, this paper uses fuzzy logic to help experts to generate these CPs.

\section{ACKNOWLEDGMENT}

The authors would like to thank the National Science

Council of the Republic of China (Taiwan) for financially supporting this research under Contract NSC 99-2221 -E-131-010-MY2.

\section{REFERENCES}

[1] F. J. Kuo, "Aquatic Ecological Risk Assessment of Dong-Gang River," Master thesis of National Kaohsiung Normal University (Taiwan), 2006.

[2] H. W. Yang, "Ecological Risk Assessment of Gao-Ping River after Downsizing of Concentrated Animal Feeding Operations," Master thesis of National Kaohsiung Normal University (Taiwan), 2006.

[3] Y. S. Lu, "The Aquatic Ecological Risk Re-assessment of Erh-Jen River" Master thesis of National Kaohsiung Normal University (Taiwan), 2006

[4] Shu-Yun Chang, "Aquatic Ecological Risk Assessment of Chiang-Chun River" Master thesis of National Kaohsiung Normal University (Taiwan), 2006.

[5] L. A. Zadeh, "From computing with numbers to computing with words- from manipulation of measurements to manipulation of perceptions," International Journal of Applied Math and Computer Science, vol.12, pp. 307-324, 2002.

[6] USEPA, Guidelines for Ecological Risk Assessment, ch. 1, pp. 2-7. 1998.

[7] J. Pearl, Probabilistic Reasoning in Intelligent Systems: Networks of Plausible Inference, Morgan Kaufmann, California, ch. 5, pp. 153-190, 1988.

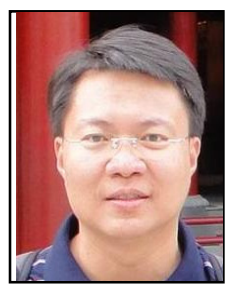

Kevin Liu received the B.S. degree in 1992 and the $\mathrm{Ph} . \mathrm{D}$. degree in 1998, both in civil engineering from National Central University, Taiwan. Currently, he is a Professor in the Department of Safety, Health and Environmental Engineering, Ming Chi University of Technology, Taiwan. His research interest is the use of decision analysis and artificial intelligence techniques to environmental management issues.

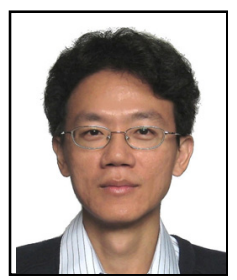

Ken Yeh received the M.S. degree in 1990 and the $\mathrm{Ph} . \mathrm{D}$. degree in 2000, both in civil engineering from National Central University, Taiwan. Currently, he is an Associate Professor in the Department of Construction Science and Technology, De-Lin Institute of Technology, Taiwan. His research interests lie generally in modeling and simulation of dynamics systems, and use of fuzzy logic techniques.

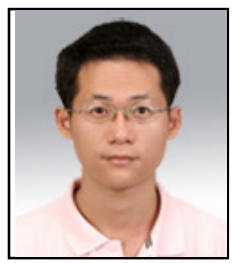

Cheng-Wu Chen is currently a Professor at the Institute of Maritime Information and Technology, National Kaohsiung Marine University. He received two Ph.D. degrees in Civil Engineering, National Central University in 2004 and Information Management, National Kaohsiung First University of Science and Technology in 2011. His research and teaching interests are in the area of applications of artificial intelligence, information technology \& system, risk analysis \& management, logistics, decision support, simulation optimization, GIS \& RS, and system control etc. forengineering and management problem solving.

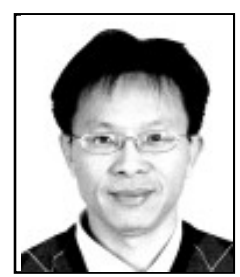

management.

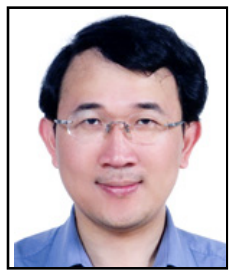

Han-Hsi Liang received the B.S. and M.S. degrees from the Department of Mechanical Engineering, National Central University, Taiwan, R.O.C., and the Ph.D. degree in Civil Engineering from National Central University, Taiwan, in 1997. He is currently an Associate Professor with the Department of Architecture, National United University, Taiwan. His research interests include decision making, green building, building material and construction

Yung-Shuen Shen received the Ph.D. degree in 1996 in chemical engineering from National Taiwan University of Science and Technology, Taiwan. Currently, he is an Associate Professor in the Holistic Education Center, Mackay Medical College, Taiwan. His research interests include incorporate environmental management and GHG issues. 